titelthema

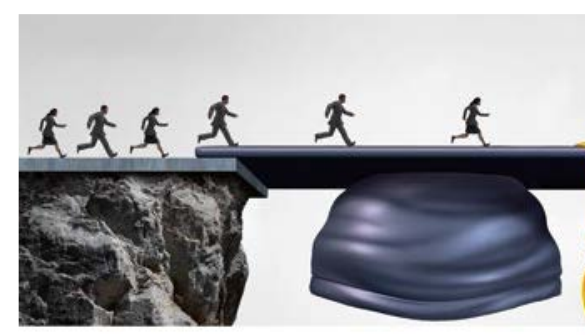

16 Zahnarzt light

Die Akademisierung der Hilfsberufe

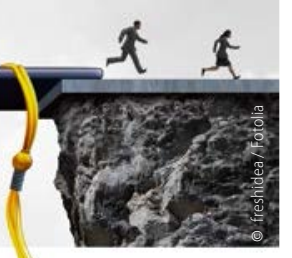

76 Myofunktionelle Diagnostik und Therapie

87 Verbundversorgungen

politik

16 Kommt der Zahnarzt light in Deutschland?

Bachelor in der Zahnmedizin

20 Zulässigkeit und Grenzen der Delegation

Übertragung zahnärztlicher Aufgaben

22 Instrumente- und Personalmangel gefährden die Lehre

Studienplatzsituation

24 Politischen Druck erzeugen

Zahnärzteschaft fordert neue Approbationsordnung

24 Überfällig

Die aktuelle Ausbildung für Studierende der Zahnmedizin basiert auf der Grundlage einer Approbationsordnung, die aus dem Jahr 1955 stammt. Deshalb fordert die Zahnärzteschaft seit langem eine zeitgemäße Neufassung, die den heutigen Anforderungen an Zahnmediziner entspricht. Da die derzeitige Bundesregierung jedoch mauert, planen die Studenten einen bundesweiten Warnstreik.

48 Überlegt

Es ist ein heiß umkämpfter Markt. Daher investieren die Hersteller von Zahnbürsten sehr viel Energie und Innovationskraft bei der Entwicklung von optimalen Modellen. Farbe, Form und Griffigkeit sind nur einige Merkmale, auf die es zu beachten gilt. Die Journalistin Dorothee Kammel hat hinter die Kulissen geblickt.

\section{1 Überzeugt}

So viele Zahnärzte auf einmal hat es in Ischgl wohl noch nie gegeben. Nach 47 Jahren in Davos fand der Winterkongress des Freien Verbandes Deutscher Zahnärzte (FVDZ) erstmals in dem Tiroler Wintersportort statt und wurde ein voller Erfolg. 
25 Neue Approbationsordnung überfällig

Fragen an die Bundeszahnärztekammer

26 Mundgesundheit von Migranten

Zahnärztliches Gipfeltreffen

27 Zahnärzte helfen Zahnärzten

28 Abgekoppelt von der wirtschaftlichen Entwicklung

Zwischenruf zu den Gebührenordnungen

30 „Zurück zur Parität“

Gesundheitspolitische Diskussionsrunde

landesverbände

32 Einladung zur Landesversammlung

Sachsen-Anhalt

33 Einladung zur Landesversammlung Sachsen

33 Einladung zur Landesversammlung Rheinland-Pfalz

34 Tatort Zahnarztpraxis Berlin

35 Bayerische Götterdämmerung Bayern

recht | steuern | wirtschaft

38 Nachlass früh und durchdacht regeln Testament mit Tücken

40 Versicherung mit flexiblem Auszahlungskonzept Konkret

42 Das Kapitalkonto - ein unbekanntes Wesen Steuertipp

46 Bewertungsportale im Internet Rechtsurteil

47 Alles, was Recht ist report

48 Eine Zahnbürste ist eine Zahnbürste ist eine Zahnbürste

Umkämpfter Markt mit hohem Innovationsdruck

praxis

52 Besondere Regelungen für schwangere Angestellte

Mutterschutz in der Zahnarztpraxis

54 Die analoge Berechnung des präendodontischen Aufbaus

Der gangbare Weg

56 GOZ-Tipp: Nervraubende Beihilfeeingriffe

$60 \mathrm{~L}$ - wie Liquiditätsrechnung für Berufsausübungsgesellschaften Buchhaltungs- $A B C$

62 Geeignete Reaktionen auf Patienteneinwände Rhetorik-Praxis

64 Buchrezension: Evolution

service

104 Serviceleistung für Verbandsmitglieder

rubriken

57 wissen kompakt

58 Mitgliedsantrag

66 aus der wissenschaft in die praxis

68 fvdz fortbildungstermine

94 dentalforum

97 dentalreport

106 zu guter letzt

107 Impressum
Wir freuen uns auf Ihr Feedback, auf Anregungen und Tipps. Schreiben Sie uns: Redaktion "Der Freie Zahnarzt" 